\title{
Potenciales consecuencias neurocognitivas de infección por virus respiratorio sincicial humano
}

\author{
Juan Carlos Flores, Karen Bohmwald, Janyra Espinoza, Cristina Jara, Marcela Peña, \\ Rodrigo Hoyos-Bachiloglu, Carolina Iturriaga, Alexis M. Kalergis y Arturo Borzutzky
}

\section{Potential neurocognitive consequences of infection by human respiratory syncytial virus}

Human respiratory syncytial virus (RSV) infection remains as a major cause of morbidity and mortality among pediatric population. Immune response is poor and unable to establish a long term effective protection against this virus. Of particular interest has been the description of extrapulmonary manifestations of RSV infection in liver, kidney, endocrine system, heart and brain, associated to infection of peripheral blood. In the central nervous system (CNS), recent studies in animals have suggested long term neurocognitive impairment due to a direct damage from the virus. This was prevented in rats by a recombinant BCG vaccine expressing a nucleoprotein $\mathrm{N}$ of $\mathrm{RSV}$ that produces an effective immune response against the virus, not allowing its dissemination to the CNS. These findings in animal models highlight the importance of conducting more specific studies in children affected with severe infection by RSV. Therefore, our group is currently conducting an assessment of the possible long-term cognitive impairment in children under 2 years. The results of this study could be a strong argument to continue looking for an effective method for protecting against RSV infection.

Key words: Human respiratory syncytial virus, encephalitis, encephalopathy, learning.

Palabras clave: Virus respiratorio sincicial humano, encefalitis, encefalopatía, aprendizaje.

\section{Introducción}

El virus respiratorio sincicial humano (VRS) es el principal agente causal de infecciones respiratorias agudas bajas (IRAB) en niños bajo dos años de edad (lactantes) en el mundo ${ }^{1}$. Una revisión sistemática reciente estimó que el VRS causó 3,4 millones de IRAB graves que requirieron hospitalización en niños bajo 5 años de edad, y entre 66.000 y 119.000 muertes en países en desarrollo el año 2005, los que concentran 99\% de la mortalidad por infecciones respiratorias ${ }^{2}$. Estos antecedentes muestran que la infección por VRS es uno de los principales problemas de salud pública en el mundo.

El VRS afecta a $70 \%$ de los niños durante el primer año de vida, y al cumplir dos años, prácticamente $100 \%$ de la población ha tenido contacto con el virus. Su incidencia más alta se alcanza entre los dos y cinco meses de edad, con brotes epidémicos durante los meses invernales ${ }^{3,4}$. Las manifestaciones clínicas de la infección por VRS varían desde cuadros asintomáticos hasta infecciones letales, dependiendo de la asociación a factores de riesgo como prematurez (gestación menor a 35 semanas), edad bajo seis meses, displasia broncopulmonar, cardiopatías congénitas e inmunosupresión ${ }^{5-11}$.

En Chile el VRS es la principal causa de IRAB en lactantes, con una tasa de hospitalización de $2 \%$ de los lactantes infectados 5 . La epidemia en el invierno chileno depende del virus predominante cada año, observándose que el VRS grupo B tiende a producir un aumento de casos más precoz ${ }^{5}$, y el grupo A se ha asociado con mayor gravedad de la infección ${ }^{12}$. En niños sin factores de riesgo, la mortalidad por IRAB en Chile es muy baja $(0,1 \%)$. Además, en un estudio reciente de co-infección de VRS con rinovirus, realizado en el área norte de Santiago, no se observó mayor gravedad en la presentación clínica ${ }^{5,13}$.

\section{Patogenia y respuesta inmune de la infección por VRS}

La infección por VRS ocurre a través de la inhalación del virus por vía respiratoria o a través del contacto directo con la mucosa ocular ${ }^{14}$. Un aspecto relevante de la infección causada por este virus es la re-infección. Se ha descrito en estudios epidemiológicos que aproximadamente $36 \%$ de los individuos puede reinfectarse durante el mismo brote estacional ${ }^{15-17}$. Este fenómeno se debe a una respuesta inmunológica ineficiente para la eliminación del virus, que sólo establece protección parcial contra infecciones posteriores ${ }^{18}$. Estudios en humanos han demostrado que la respuesta inicial es mediada por células epiteliales, las que promueven el
Pontificia Universidad Católica de Chile, Santiago, Chile Escuela de Medicina, División de Pediatría (JCF).

Departamento de Enfermedades Infecciosas e Inmunología Pediátrica (RHB, $\mathrm{Cl}, \mathrm{AB}$ ). Departamento de Endocrinología (AMK). Facultad de Ciencias Biológicas, Departamento de Genética Molecular y Microbiología (KB, JE, AMK)

Escuela de Psicología, Laboratorio de Neurociencias Cognitivas (C, MP).

Instituto Milenio en Inmunología e Inmunoterapia, (AMK, AB). Complejo Asistencial Dr. Sótero del Río, Santiago, Chile. Servicio de Pediatría (JCF)

Los autores no tienen conflictos de interés con este manuscrito. Financiamiento: Proyecto Interdisciplina 14/2013, Vicerectoría de Investigación, Pontificia Universidad Católica de Chile. Instituto Milenio en Inmunología e Inmunoterapia P09/016-F. Fondo de Desarrollo Científico y Tecnológico proyecto número 1150862. JE y $\mathrm{KB}$ son becarias de doctorado de CONICYT.

Recibido: 28 de marzo de 2016 Aceptado: 23 de junio de 2016

Correspondencia a: Arturo Borzutzky Schachter arturobor@med.puc.cl 
reclutamiento de células inmunes al sito de la infección. La inmunopatología mediada por VRS se caracteriza por la producción de citoquinas pro-inflamatorias que atraen a células mononucleares, principalmente neutrófilos y linfocitos, al espacio peribronquial. Se describe una respuesta inflamatoria exacerbada debido a un desbalance de linfocitos $\mathrm{Th}_{2}$ patogénicos, además de una reducción en la actividad de linfocitos $T$ citotóxicos ${ }^{19,20}$.

Otro factor relevante en la patología causada por VRS es el estado inmunológico del individuo; se ha descrito que hace variar la presentación clínica. En pacientes inmunocompetentes la presentación clínica más común es obstrucción de la vía aérea, sin afectación importante a nivel alveolar. Por el contrario, en inmunodeficientes se ha observado mayor frecuencia de neumonía, con infiltrados celulares en el parénquima pulmonar y menor frecuencia de sibilancias ${ }^{21}$.

\section{Manifestaciones extra-pulmonares de infección por VRS grave}

Durante la última década se han reportado diversas manifestaciones extra-pulmonares asociadas a la infección por VRS, entre las cuales están alteraciones hepáticas ${ }^{22,23}$, renales $^{24}$, endocrinas ${ }^{22,25}$, cardiovasculares ${ }^{26,28}$ y del sistema nervioso central (SNC) $)^{22,29-31}$. El compromiso de estos dos últimos sistemas podría explicar la asociación del virus con muerte súbita en lactantes menores ${ }^{22,26}$.

Se ha descrito el compromiso hemodinámico con hipotensión arterial, necesidad de fármacos vasoactivos, daño miocárdico con elevación de enzimas cardíacas y arritmias ventriculares y supraventriculares. La presencia del virus en miocardio ha sido demostrada con métodos de cultivo y reacción de polimerasa en cadena (RPC $)^{26}$.

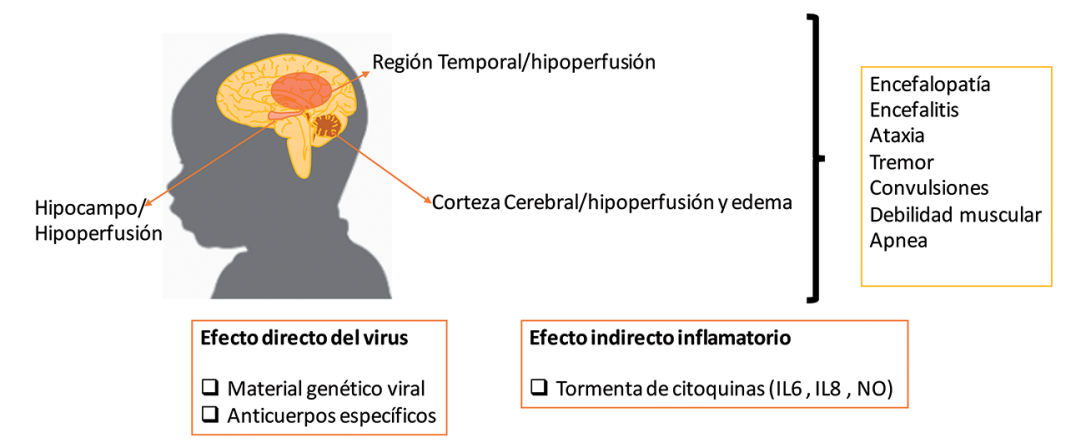

Figura 1. Hallazgos clínicos de alteraciones en el sistema nervioso central asociadas a la infección con VRS. La figura muestra las zonas del cerebro que estarían afectadas por la infección con VRS, así como la sintomatología clínica asociada. Además se destacan los efectos directos e indirectos del VRS en el SNC.
Alrededor de $50 \%$ de los niños que requieren apoyo ventilatorio derivado de bronquiolitis grave presentan elevación de transaminasas, hecho que se asoció a un mayor número de días en ventilación mecánica. Al igual que en el compromiso cardíaco, el virus logró ser cultivado desde una muestra de biopsia hepática en un paciente inmunocompetente ${ }^{26}$.

La infección por VRS también ha sido asociada con manifestaciones renales. Se ha encontrado material genético y antígenos virales por RPC en muestra de orina de un paciente pediátrico con síndrome nefrótico, que tuviera una buena respuesta a corticosteroides ${ }^{24}$.

El efecto endocrino más importante es la hiponatremia producida por secreción inapropiada de hormona antidiurética, descrita en 33\% de los pacientes que ingresaron a unidad de cuidados intensivos con infección grave por VRS. Las convulsiones por hiponatremia se asociaron a la administración de soluciones hipotónicas de mantención. Otras alteraciones hormonales descritas en pacientes en ventilación mecánica son concentraciones aumentadas de prolactina y hormona de crecimiento, y una disminución de leptina e IGF- ${ }^{26}$.

La diseminación sistémica de VRS ha sido asociada a la infección de células inmunes en el torrente sanguíneo. Reportes en modelos animales (ratones) y humanos (lactantes) han demostrado la detección de este patógeno en sangre, mediante técnica de RT-PCR ${ }^{32,33}$. Esta evidencia sugiere que VRS es capaz de diseminarse a órganos distantes utilizando la vía hematógena ${ }^{32}$.

\section{Compromiso de sistema nervioso central}

Alrededor de $2 \%$ de los casos de bronquiolitis por VRS reportan síntomas derivados de compromiso del $\mathrm{SNC}^{30,31}$. Estudios en humanos han encontrado la presencia de ARN viral y anticuerpos específicos contra VRS en líquido cefalorraquídeo (LCR) ${ }^{34}$. Las áreas del SNC que se han descrito afectadas por la infección por VRS incluyen hipocampo, troncoencéfalo, corteza cerebelar y región temporal (Figura 1) ${ }^{35-37}$. Estos hallazgos han sido asociados a síntomas neurológicos como convulsiones, apneas centrales, letargia, alteraciones de la deglución, estrabismo, anormalidades del tono y encefalopatía ${ }^{22,29-31,35,38,39}$.

La etiología de la encefalopatía causada por la infección con VRS es aún desconocida. Estudios en muestras de LCR de pacientes con complicaciones neurológicas han mostrado concentraciones elevadas de citoquinas pro-inflamatorias, tales como IL-6, IL-8 y óxido nítrico, sugiriendo que una exacerbada producción de estos mediadores inflamatorios podría estar involucrada en la encefalopatía causada por $\operatorname{VRS}^{30,36}$. Morichi y cols., correlacionan además las concentraciones elevadas de IL-6 y brain-derived neurotrophic factor (BDNF) con 
secuelas neurológicas evaluadas por puntaje estandarizado (Pediatric Cerebral Performance Categories) a los seis meses de ocurrida el alta, en una serie pequeña de 11 pacientes con encefalopatía por VRS ${ }^{40}$. Otra investigación reciente de Erez y cols., apoya la hipótesis inflamatoria como causa del compromiso de SNC debido a que las manifestaciones clínicas se presentan aún en ausencia de ARN viral en el $\mathrm{LCR}^{41}$. En dicho estudio, se estudiaron lactantes con infección confirmada por VRS a quienes se realizó punción lumbar por síndrome febril en niños bajo 6 semanas de edad, por sospecha de infección bacteriana o compromiso de SNC. Se separó a los pacientes en dos grupos de acuerdo a la presencia o ausencia de manifestaciones neurológicas en la presentación clínica (apneas, convulsiones y encefalopatía). Se evaluó presencia de ARN viral en el LCR mediante RPC para VRS, virus influenza, parainfluenza, metapneumovirus humano, enterovirus, adenovirus y parechovirus ${ }^{41}$. Todas las muestras resultaron negativas para los agentes infecciosos estudiados, en ambos grupos de pacientes. Los autores sugieren que probablemente hay mecanismos inmunológicos involucrados en las manifestaciones neurológicas asociadas a VRS, que en esta serie correspondieron a apnea y encefalopatía (compromiso de conciencia).

Con respecto a la gravedad, es importante destacar que sólo 50\% del grupo de pacientes con síntomas neurológicos, y ninguno en el grupo control, requirió ventilación mecánica durante la hospitalización ${ }^{41}$. Esta información es relevante debido a que en la mayoría de los estudios que han reportado presencia de VRS en LCR se han incluido pacientes con bronquiolitis grave.

Actualmente el número de casos reportados de encefalopatía asociada a infección grave por VRS ha ido en aumento, recalcando la importancia de conocer más en detalle este fenómeno. No se conoce aún con exactitud la fisiopatología que explica esta presentación clínica, su mecanismo de entrada al SNC, localización y forma de diseminación ${ }^{30}$. Nuestro grupo, (Espinoza J. y cols.), evaluó las características del compromiso neurológico en modelos animales de infección por VRS (ratones $\mathrm{Balb} / \mathrm{cJ}$ y ratas Sprague-Dawley), con resultados que
A

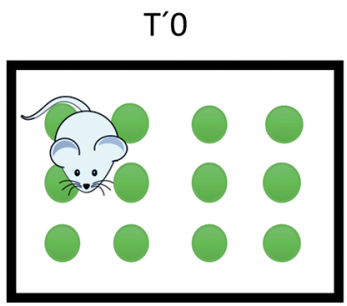

Test Marble Burying

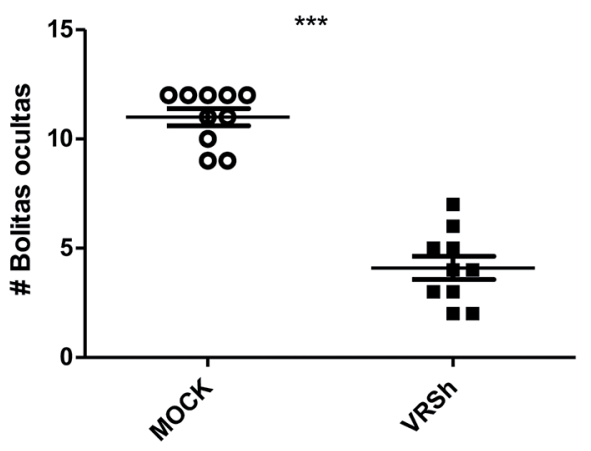

T'30

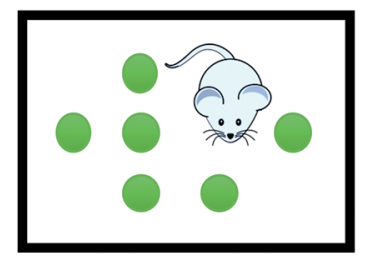

B

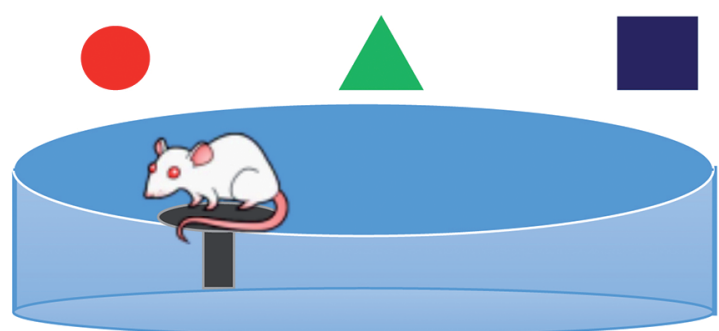

Test Morris Water Maze

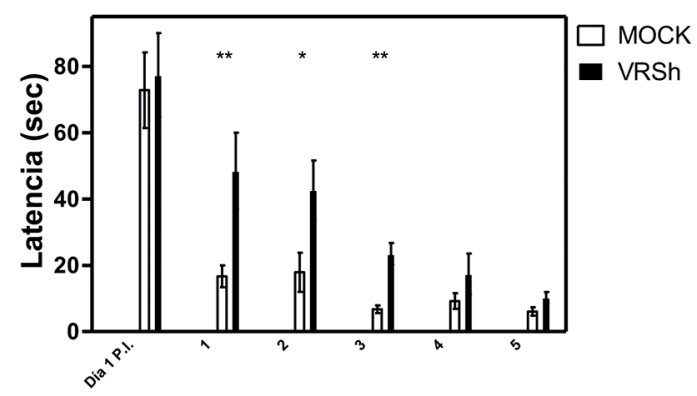

Días de Prueba

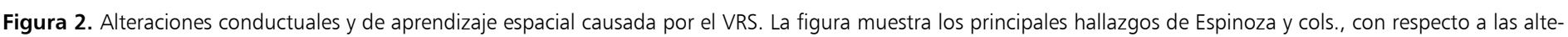

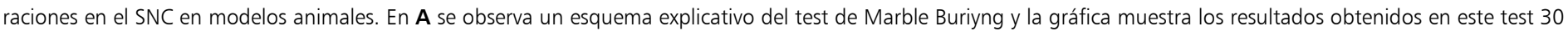

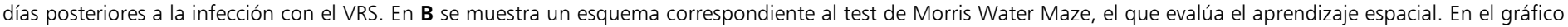
se puede observar los resultados obtenidos 30 días después de finalizar la infección por VRS. 
apoyan la infección directa por parte del virus en el SNC y que permiten entender los mecanismos por los cuales se produce ${ }^{42}$. Después de inoculación nasal de VRS se realizó un detallado monitoreo de la progresión de la enfermedad, encontrándose un patrón de compromiso focal en el SNC, con mayor presencia de ARN y proteínas del virus en las áreas relacionadas a la producción de LCR, bulbo olfatorio y en aquellas con mayor irrigación sanguínea. Estos datos sugieren que el virus posee un neurotropismo e ingresa al SNC por dos vías distintas: hematógena, contenido en leucocitos infectados, y a través de nervio olfatorio ${ }^{42}$.

\section{Alteraciones cognitivas y de conducta posteriores a la infección aguda por VRS}

En el estudio realizado por Espinoza J. y cols., además se encontraron proteínas virales en la zona del hipocampo, la que es muy importante para procesos $\operatorname{cognitivos}^{42}$. Por este motivo, se evaluó si los animales infectados con VRS presentaron algún tipo de alteración en aprendizaje y conducta. Los animales fueron evaluados a los 30 días después de la infección con VRS en términos del aprendizaje espacial utilizando el test de Morris Water Maze $(\mathrm{MWM})^{42}$. Los resultados obtenidos en este test mostraron que las ratas que fueron infectadas con VRS presentan problemas cognitivos con capacidad de aprendizaje alterada (Figura 2). Por otra parte, se evaluó la conducta, tanto de ratas como ratones, utilizando el test de Marble Burying (MB) a 30 días de terminada la infección con VRS. Los resultados mostraron que los animales infectados presentaban alteraciones en el comportamiento al ser comparados con animales control ${ }^{42}$. Estas alteraciones lograron ser prevenidas utilizando inmunización con vacuna recombinante BCG que expresa la nucleoproteína $\mathrm{N}$ de VRS, la cual es capaz de inducir inmunidad efectiva, impidiendo la diseminación del virus hacia el SNC y, en consecuencia, evitando las alteraciones anteriormente $\operatorname{descritas}^{42}$.

\section{Perspectivas futuras para investigación y desarrollo de vacuna contra VRS}

Los antecedentes mencionados en este artículo dan cuenta de la importancia de estudiar en humanos si el VRS es capaz de causar alteraciones cognitivas como las descritas en animales. Otro aspecto relevante a destacar es la necesidad de desarrollar una vacuna que sea efectiva y de ese modo prevenir las graves consecuencias neurológicas que puede provocar la infección por VRS. No se han dado a conocer, a la fecha de envío de este artículo, estudios en los cuales se evalúe si el VRS es capaz de producir alteraciones en el aprendizaje a largo plazo. A raíz de estos hallazgos en animales, en nuestro grupo surgió el interés de realizar una investigación, actualmente en curso, que busca caracterizar cualitativa y cuantitativamente si la infección grave por VRS produce consecuencias cognitivas en niños, en una evaluación prospectiva.

Antes de cumplir un año, los niños han avanzado notablemente en el desarrollo de destrezas cognitivas cruciales para la vida, tales como la lengua materna ${ }^{43,44}$, cognición numérica ${ }^{45,46}$ y cognición social ${ }^{47}$. Estudios de nuestro grupo en esta área han contribuido a caracterizar el rol de la biología y la experiencia en el logro de estas habilidades, tanto en niños sanos como en riesgo cognitivo ${ }^{44,48,49}$. Desde esta perspectiva, el estudio en desarrollo evalúa el impacto de la infección grave por VRS en la adquisición de hitos del desarrollo lingüístico, numérico y social por medio de pruebas estandarizadas realizadas 1 , 3 y 6 meses después de haberse resuelto el cuadro clínico. Para estimar la especificidad del compromiso cognitivo asociado a VRS se realizará una comparación con niños sanos no afectados por VRS grave.

Esta área de estudio, sin duda, es de alto interés para las ciencias médicas y para la sociedad en general, debido a las potenciales consecuencias en el neurodesarrollo de una enfermedad de alto impacto epidemiológico y para la cual no existe en la actualidad un método costoefectivo para tratar o prevenir la infección. Conocer si VRS produce alteraciones neurocognitivas en humanos podría permitir además el desarrollo de protocolos de diagnóstico e intervención precoz para tratar oportunamente los problemas derivados de la infección grave por este virus.

Agradecimientos. Los autores agradecen el apoyo del Proyecto Interdisciplina de la Vice-Rectoría de Investigación UC, el Instituto Milenio en Inmunología e Inmunoterapia, Proyecto FONDECYT 1150862 y de la Comisión Nacional de Investigación Científica y Tecnológica de Chile.

\section{Resumen}

La infección por virus respiratorio sincicial humano (VRS) es una de las principales causas de morbimortalidad en población pediátrica. La respuesta inmune generada contra VRS es poco eficiente para su eliminación y logra establecer sólo protección parcial contra infecciones posteriores. De especial interés en los últimos años ha sido la descripción de manifestaciones extra-pulmonares de la infección por VRS en hígado, riñón, sistema endocrino, corazón y cerebro. A nivel de sistema nervioso central (SNC), estudios recientes en modelos animales han sugerido problemas neurocognitivos a largo plazo derivados de un daño directo del virus en el cerebro. Este 
daño logró ser prevenido con vacuna experimental BCG recombinante, que expresa la nucleoproteína $\mathrm{N}$ de VRS e induce inmunidad efectiva, impidiendo la diseminación del virus hacia el SNC. Estos hallazgos en modelo animal han dado cuenta de la importancia de efectuar estudios más detallados en niños afectados por VRS grave. Por tal motivo, actualmente se está realizando una evaluación de la posible alteración cognitiva a largo plazo en niños bajo dos años de edad por parte de nuestro grupo. Los resultados de este estudio podrían significar un argumento muy importante para continuar en la búsqueda de un método efectivo de protección contra esta infección.

\section{Referencias bibliográficas}

1.- Hall C B, Weinberg G A, Iwane M K, Blumkin A K, Edwards K M, Staat M A, et al. The burden of respiratory syncytial virus infection in young children. N Engl J Med 2009; 360: 588-98.

2.- Nair H, Nokes D J, Gessner B D, Dherani M, Madhi S A, Singleton R J, et al. Global burden of acute lower respiratory infections due to respiratory syncytial virus in young children: a systematic review and meta-analysis. Lancet 2010; 375: 1545-55.

3.- Baker K A, Ryan M E. RSV infection in infants and young children. What's new in diagnosis, treatment, and prevention? Postgrad Med 1999; 106: 97-99, 103-4, 107-8 passim.

4.- Henderson F W, Collier A M, Clyde W A, Jr., Denny F W. Respiratory-syncytial-virus infections, reinfections and immunity. A prospective, longitudinal study in young children. N Engl J Med. 1979; 300: 530-4.

5.- Avendaño L F, Palomino M A, Larranaga C. Surveillance for respiratory syncytial virus in infants hospitalized for acute lower respiratory infection in Chile (1989 to 2000). J Clin Microbiol 2003; 41: 4879-82.

6.- Avendaño L F P J, Padilla C, Palomino M A. Impacto en salud infantil del invierno 2002: disociación entre factores ambientales y virus respiratorio sincicial, en Santiago. Rev Med Chile 2003; 902-8.

7.- Meert K, Heidemann S, Lieh-Lai M, Sarnaik A P. Clinical characteristics of respiratory syncytial virus infections in healthy versus previously compromised host. Pediatr Pulmonol 1989; 7: 167-70.

8.- Palomino M A, Avendaño L. Infecciones respiratorias por VRS y adenovirus en Chile: qué hemos aprendido en 14 años de vigilancia epidemiológica. Rev Pediatría (Santiago) 2003; 43: 38-47.

9.- Wang E E, Law B J, Robinson J L, Dobson S, al Jumaah S, Stephens D, et al. PICNIC (Pediatric Investigators Collaborative Network on Infections in Canada) study of the role of age and respiratory syncytial virus neutralizing antibody on respiratory syncytial virus illness in patients with underlying heart or lung disease. Pediatrics 1997; 99: E9.

10.- DeVincenzo J P, El Saleeby C M, Bush A J. Respiratory syncytial virus load predicts disease severity in previously healthy infants. J Infect Dis 2005; 191: 1861-8.

11.- Wang E E, Law B J, Stephens D. Pediatric Investigators Collaborative Network on Infections in Canada (PICNIC) prospective study of risk factors and outcomes in patients hospitalized with respiratory syncytial viral lower respiratory tract infection. J Pediatr 1995; 126: 212-9.

12.- Moore M L, Stokes K L, Hartert T V. The impact of viral genotype on pathogenesis and disease severity: respiratory syncytial virus and human rhinoviruses. Curr Opin Immunol 2013; 25: 761-8.

13.- Luchsinger V, Ampuero S, Palomino M A, Chnaiderman J, Levican J, Gaggero A, et al. Comparison of virological profiles of respiratory syncytial virus and rhinovirus in acute lower tract respiratory infections in very young Chilean infants, according to their clinical outcome. J Clin Virol 2014; 61: 138-44.

14.- Hall C B, Douglas R G, Jr, Schnabel K C, Geiman J M. Infectivity of respiratory syncytial virus by various routes of inoculation. Infect Immun 1981; 33: 779-83.

15.- Dudas R A, Karron R A. Respiratory syncytial virus vaccines. Clin Microbiol Rev 1998; 11: 430-9.

16.- Paramore L C, Ciuryla V, Ciesla G, Liu L. Economic impact of respiratory syncytial virus-related illness in the US: an analysis of national databases. Pharmacoeconomics 2004; 22: $275-84$.

17.- Storey S. Respiratory syncytial virus market. Nat Rev Drug Discov 2010; 9: 15-6.

18.- Bueno S M, González P A, Pacheco R, Leiva E D, Cautivo K M, Tobar H E, et al. Host immunity during RSV pathogenesis. Int Immunopharmacol 2008; 8: 1320-9.

19.- Gut W, Pancer K, Abramczuk E, Czescik A, Dunal-Szczepaniak M, Lipka B, et al. RSV respiratory infection in children under 5 y.o.dynamics of the immune response Th1/Th2 and IgE Przegl. Epidemiol 2013; 67: 17-22, 105-9.

20.- Guo-Parke H, Canning P, Douglas I, Villenave R, Heaney L G, Coyle P V, et al. Relative respiratory syncytial virus cytopathogenesis in upper and lower respiratory tract epithelium. Am J Respir Crit Care Med 2013; 188: 842-51.

21.- Whimbey E, Couch R B, Englund J A, Andreeff M, Goodrich J M, Raad, II, et al.
Respiratory syncytial virus pneumonia in hospitalized adult patients with leukemia. Clin Infect Dis 1995; 21: 376-9.

22.- Eisenhut M. Extrapulmonary manifestations of severe respiratory syncytial virus infection--a systematic review. Crit Care 2006; 10: R107.

23.- Kirin B K, Topic R Z, Dodig S. Hepatitis during respiratory syncytial virus infection-a case report. Biochem Med (Zagreb) 2013; 23: 112-6.

24.- Wu J, Wang Z, Wang D. Respiratory virus expression in renal tissues and urine of children with steroid responsive simple nephrotic syndrome. Sichuan Da Xue Xue Bao Yi Xue Ban 2007; 38: 969-72.

25.- Hanna S, Tibby S M, Durward A, Murdoch I A. Incidence of hyponatraemia and hyponatraemic seizures in severe respiratory syncytial virus bronchiolitis Acta Paediatr. 2003; 92: 430-4.

26.- Eisenhut M. Extrapulmonary manifestations of severe RSV bronchiolitis Lancet. 2006; 368 : 988.

27.- Menchise A. Myocarditis in the setting of RSV bronchiolitis. Fetal Pediatr Pathol 2011; 30: 64-8.

28.- Puchkov G F, Min'kovich B M. Respiratory syncytial infection in a child complicated by interstitial myocarditis with fatal outcome. Arkh Patol 1972; 34: 70-3.

29.- Millichap J J, Wainwright M S. Neurological complications of respiratory syncytial virus infection: case series and review of literature. J Child Neurol 2009; 24: 1499-503.

30.- Morichi S, Kawashima H, Ioi H, Yamanaka G, Kashiwagi Y, Hoshika A, et al. Classification of acute encephalopathy in respiratory syncytial virus infection J Infect Chemother 2011; 17: 776-81.

31.- Sweetman L L, Ng Y T, Butler I J, Bodensteiner J B. Neurologic complications associated with respiratory syncytial virus. Pediatr Neurol 2005; 32: 307-10.

32.- O'Donnell D R, McGarvey M J, Tully J M, Balfour-Lynn I M, Openshaw P J. Respiratory syncytial virus RNA in cells from the peripheral blood during acute infection. J Pediatr 1998; 133: 272-4.

33.- Torres J P, Gómez A M, Khokhar S, Bhoj V G, Tagliabue C, Chang M L, et al. Respiratory syncytial virus (RSV) RNA loads in peripheral blood correlates with disease severity in mice Respir Res 2010; 11: 125.

34.- Zlateva K T, Van Ranst M. Detection of 
subgroup B respiratory syncytial virus in the cerebrospinal fluid of a patient with respiratory syncytial virus pneumonia Pediatr Infect Dis J 2004; 23: 1065-6.

35.- Hirayama K, Sakazaki H, Murakami S, Yonezawa S, Fujimoto K, Seto T, et al. Sequential MRI, SPECT and PET in respiratory syncytial virus encephalitis. Pediatr Radiol 1999; 29: 282-6.

36.- Miyamoto K, Fujisawa M, Hozumi H, Tsuboi T, Kuwashima S, Hirao J, et al. Systemic inflammatory response syndrome and prolonged hypoperfusion lesions in an infant with respiratory syncytial virus encephalopathy. $\mathrm{J}$ Infect Chemother 2013; 19: 978-82.

37.- Park A, Suh S I, Son G R, Lee Y H, Seo H S, Eun B L, et al. Respiratory syncytial virusrelated encephalitis: magnetic resonance imaging findings with diffusion-weighted study. Neuroradiology 2014; 56: 163-8.

38.- Eisenhut M. Cerebral involvement in respiratory syncytial virus disease. Brain Dev 2007; 29 : 454
39.- Kho N, Kerrigan J F, Tong T, Browne R, Knilans J. Respiratory syncytial virus infection and neurologic abnormalities: retrospective cohort study. J Child Neurol 2004; 19: 859-64.

40.- Morichi S, Morishita N, Ishida Y, Oana S, Yamanaka G, Kashiwagi Y, et al. Examination of neurological prognostic markers in patients with respiratory syncytial virus-associated encephalopathy. Int J Neurosci 2016: 1-6.

41.- Erez D L, Yarden-Bilavsky H, Mendelson E, Yuhas Y, Ashkenazi S, Nahum E, et al. Apnea induced by respiratory syncytial virus infection is not associated with viral invasion of the central nervous system. Pediatr Infect Dis J 2014; 33: 880-1.

42.- Espinoza J A, Bohmwald K, Cespedes P F, Gómez R S, Riquelme S A, Cortés C M, et al. Impaired learning resulting from respiratory syncytial virus infection. Proc Natl Acad Sci U S A. 2013; 110: 9112-7.

43.- Pena M, Maki A, Kovacic D, DehaeneLambertz G, Koizumi H, Bouquet F, et al. Sounds and silence: an optical topography study of language recognition at birth. Proc Natl Acad Sci USA 2003; 100: 11702-5.

44.- Pena M, Werker J F, Dehaene-Lambertz G. Earlier speech exposure does not accelerate speech acquisition. J Neurosci 2012; 32: 11159-63.

45.- Gervain J, Mehler J. Speech perception and language acquisition in the first year of life. Annu Rev Psychol 2010; 61: 191-218.

46.- Pena M, Pittaluga E, Mehler J. Language acquisition in premature and full-term infants. Proc Natl Acad Sci USA 2010; 107: 3823-8.

47.- Cordes S, Brannon E M. Quantitative competencies in infancy Dev Sci 2008; 11: 803-8

48.- Feigenson L, Dehaene S, Spelke E. Core systems of number. Trends Cogn Sci 2004; 8: 307-14.

49.- Gergely G, Csibra G. Natural pedagogy. In Navigating the Social World: What Infants, Chidren, and Other Species Can Teach Us: Oxford University Press; 2013. 